\title{
A New Computer-based Ferromagnetic Metal Detector for Security Applications
}

\author{
Francis K. Murori \\ Department of Physics \\ Kenyatta University, PO BOX \\ 40844, Nairobi, Kenya
}

\author{
Elijah Mwangi \\ School of Engineering \\ University of Nairobi, PO BOX \\ 30197, Nairobi, Kenya
}

\author{
Patrick M. Karimi \\ Institute Energy Studies and \\ Research \\ Kenya Power, PO BOX 30099 \\ Nairobi, Kenya
}

\begin{abstract}
The need for detecting and locating concealed metallic objects and contraband items increases in importance as the issues of security becomes of great concern. In the field of metal detectors, the research is being geared towards the production of detectors with fast settling times and high discriminating ability. This paper proposes a method for designing a computer-based ferromagnetic metal detector which can be employed for security applications. The LabVIEW software has been used to program the detector. It is a novel programming language from National Instruments that is robust, highly parallel and eliminates most of syntactical details associated with text based programming languages. Because of this, the development time of the detector is reduced by a significant factor. A recognition algorithm has also been designed to discriminate between ferromagnetic items by their mass and size. To improve the settling time of the detector, components with fast response times have been chosen, making the detector's settling time to be approximately $1.1 \mathrm{sec}$. In addition, the screening process has been made more convenient by connecting the detector to a remote computer through data socket networking. The remote computer enables the detector to be monitored remotely in real time and also acts as a central database where the screening information is sent for further storage.
\end{abstract}

The system hardware comprises of ten fluxgate magnetic field sensors (FLC 100) with response time of $1 \mathrm{sec}$ and sensitivity of $1 \mathrm{~V} / 50 \mu \mathrm{T}$, a wooden frame in form of a portal, connecting cables, USB DAQ 2833, USB video camera, wireless network adapter and two laptop computers.

\section{Keywords}

Magnetic field sensors (FLC 100), LabVIEW programming, USB Video camera, USB DAQ 2833, Data socket Networking

\section{INTRODUCTION}

The rise in global insecurity has increased the need to ensure that the public are protected at all times. The frequent cases of terrorism attacks have awakened fear and sense of insecurity in places where peace once reigned. In addition, the vice of arms smuggling have ended up leaving a lot of arms in the hands of civilians, hence it is becoming a serious challenge to the authorities on how to counter these problems. In response to these problems, the screening for the weapons and contraband items is routinely being done at the entrances of places such as airports, embassies, churches, night clubs, amusement parks and other public places which may be vulnerable to threats or attacks. Assault weapons such as guns, grenades and knives are routinely made from ferromagnetic materials [1]. These are materials whose magnetic moments tend to align in the direction of an ambient magnetic field. Example of ferromagnetic materials includes iron, nickel, cobalt, and various alloys thereof.

There are a number of reasons why the screening process remains relatively slow (i.e. low throughput), these are;

i) False alarms - these results from non-threat item such watches, shoe shanks, belt buckles, earrings, phones etc. In some cases a subject is required to surrender these items to an attendant for visual inspection. This slows down the whole process.

ii) Poor settling times - the detector takes too long to process the data after a subject has passed through the detector.

According to Sigrist [2], the field of metal detectors has been confronted with lack of adequate scientific and technical literature. The field is therefore still evolving and has not established itself well in the literature. Due to the aforementioned problem, the citations given in this section of the recent advancements made in this field have been taken from other applications and not necessarily from security.

Burns [3] designed a computer based ferromagnetic metal detector for vehicle detection, classification and speed measurement referred to as 'In-situ vehicle classification'. The vehicles' components have high percentage of ferromagnetic material. Inductive loop detectors and magneto resistive sensors were used to detect changes in magnetic fields once the vehicle passes. The magnetic data collected could be used to provide a unique mapping of vehicle configuration. Data collection and analysis was done by MATLAB.

Bjerrum [4] designed a metal detector for use in food industries. It was operated by radiating active magnetic fields. When the electromagnetic field falls on the metal target, it gets distorted thus altering its amplitude, phase and frequency. The amplitude and phase differences are extracted from the signal received from metal detector head coil. To classify various metallic items, Covariance and Bayesian classifiers were used. Data analysis was based on both MATLAB and C programming.

Kalyn [5] designed a ferromagnetic metal detector for indoor localization using earth's magnetic fields. It consists of locating oneself inside buildings as GPS does not work indoors due multipath reflection and signal blockage. This method exploits the indoor ambient magnetic fields resulting from anomalies in earth's magnetic fields caused by pillars, doors and elevators which are ferromagnetic in nature. By observing uniqueness in magnetic signatures collected from different buildings, the magnetic maps of buildings can be developed and used to locate and navigate subjects indoors. The localization application was developed solely on the smart phone where the magnetic field sensor ( Hall effect 
sensor) in the smart phone is used to detect magnetic field disturbances. The application was written in Java language using Android Application Programming Interface (API).

Sajjad [6] designed a computer based metal detector for localization and identification of ferromagnetic items. The target detection, identification and sequential localization were accomplished by using an algorithm referred to as Minimum Euclidean Distance (MED). Continuous movements of ferromagnetic items were monitored and the magnetic field changes measured by the sensor were used to obtain such information as target direction and the type of target detected. The data analysis was done using MATLAB.

Yin [7] et al. designed a microcontroller based metal detector using Mikro $\mathrm{C}$ programming language. When a metal is brought near the sensing coil of the detector the output voltage from the sensing coil increases and it is compared to a reference (threshold) voltage. No recognition algorithm was designed but voltage thresholds were used to discriminate between ferrous and non-ferrous metals.

In the field of metal detectors, the research is being geared towards production of detectors with fast settling times and high discriminating ability [8]. In this paper, we propose a computer based ferromagnetic metal detector for security applications programmed using LabVIEW software. The existing detectors are mostly designed using text based programming languages; not much has been done in using LabVIEW as programming language. It's a novel programming language from National Instruments which is robust, highly parallel and eliminates most of syntactical details associated with text based programming languages[9][10]. Because of this, the development time of the detector is reduced by a significant factor. A recognition algorithm has also been designed to discriminate between ferromagnetic items by their mass and size. A significant advantage of LabVIEW software is its potential to create recognition algorithms. To improve the settling time of the detector, components with fast response times have been chosen, these are, magnetic field sensors (FLC 100) with response time of approximately $1 \mathrm{sec}$, USB DAQ (2833) with an ADC of response time of $1.6 \mu$ s and USB camera with response time of $50 \mathrm{~ms}$. This makes the overall detectors settling time be less than $1.1 \mathrm{sec}$.

The rest of this paper is organized as follows: In section 2, the theory of metal detection is given. Section 3 is a presentation of the proposed detector while section 4 gives a presentation of the experimental results obtained by moving a ferromagnetic item towards a magnetic sensor as well as the results from the front panel of the detector. Section 5 gives a conclusion and recommendation for further work.

\section{THEORETICAL BACKGROUND 2.1 Ferromagnetic Objects in Earth's Magnetic Field.}

When a ferromagnetic object is placed in earth's magnetic field it disturbs the earth's magnetic field at that region. The resulting disturbances are referred to as magnetic anomalies and they are measured by a magnetic field sensor. The magnetic field disturbance $B$, at a distance $r$, caused by a ferromagnetic item can be modelled by the equation (1) below [11].

$$
\vec{B}=\frac{\mu_{0}}{4 \pi r^{3}}[3 \vec{r}(\vec{r} \cdot m)-m]
$$

- $\overrightarrow{\mathrm{B}}$ is the magnetic field induction
- $\mu_{0}=4 \pi \times 10-7$ is the permeability of free space

- $\quad$ ris the distance between the ferromagnetic item and the observation point (sensor).

- $\quad \overrightarrow{\mathrm{r}}$ is the unit vector in $\mathrm{r}$ direction

- $\mathrm{m}$ is the magnetic moment

In earth's magnetic field, a ferromagnetic object can exhibit either a dipole or a monopole response. The type of response displayed depends on the orientation and geometry of the ferromagnetic object. Equation (1) predicts that the magnetic field will reduce at a rate of $1 / r^{3}$, this is true for dipole response. On the other hand, if an item displays monopole response its magnetic field will reduce at rate of $1 / r^{2}$ [11]

\subsection{Electromagnetic Induction (EMI) Metal Detection Systems}

An EMI system operates by periodically broadcasting a series of electromagnetic pulses, usually in the $\mathrm{kHz}$ range. It consists of two coils, that is the transmitter and the receiver coil. The transmitted electromagnetic pulse induces eddy currents on the surface of the metallic object within the sensing area. The eddy currents in turn induce a voltage signal on the receiver coil. If the transmitter/primary coil transmits a sine wave with single frequency $\omega$ and amplitude $A_{p}$, that is,

$$
V_{\text {prim }}=A_{P} \sin \omega t
$$

Then the secondary (received) signal will be given by,

$$
V_{\mathrm{sec}}(t)=A_{\mathrm{sec}} \sin (\omega t+\varnothing)
$$

It can also be noted that, the amplitude and the phase of the received signal have been altered. These parameters depend on the type, size, and shape of the metallic object being detected. In some cases the frequency, $\omega$ may also be altered [12]. Because there is external generation of electromagnetic field, these systems are said to be electromagnetically active.

Although EMI metal detection systems have been used for decades, they have some limitations, these are;

1. There is high power consumption due to generation of electromagnetic signals

2. The generated electromagnetic fields can interfere with the cardiac pacemakers which are worn by persons with heart problems [8]

The above limitations may be overcome by the use of passive metal detection system.

\subsection{Passive Metal Detection System}

The metal detection system based on passive method utilizes the earth's magnetic fields. In these systems, electromagnetic fields or pulses are not generated when a person is being screened; instead, perturbations of earth's magnetic fields are detected. It is for this reason that they are said to be passive. The disturbances caused by ferromagnetic items are usually very small and they are detected by a sensitive magnetic field sensor. The shape of the signal generated depends on such factors as, the type of object, its distance from the sensor and its orientation in earth's field among others. Each ferromagnetic material has a unique magnetic signature in earth's magnetic field. The uniqueness of these magnetic signatures can be used to identify and classify ferromagnetic objects in earth's magnetic field. The main advantage of passive metal detection system is that, the earth's magnetic field does not interfere with nearby electronic devices such as heart pacemakers [8]. Therefore, persons with heart 
pacemakers may be screened without any risk. In addition, since there is no generation of EM pulses, the power consumption is low [13].

\subsection{Computer-Based Metal Detection System}

A metal detector can be interfaced to a microcontroller, microprocessor or to a computer to enhance its efficiency. One advantage of computer based metal detector is that it can be 'trained'. In this case the magnetic signatures of unknown ferromagnetic items are viewed on the computer screen, analyzed, features extracted and classified and then added to the database thus allowing the system to become 'smarter' over time. Training Computer based metal detector is done by means of recognition algorithms such as Artificial Neural Networks (ANN) and Bayesian classifiers among others. In addition, in a computer based metal detector the results can be displayed in various human-readable formats since this only requires reprogramming the computer [8].

A further advantage of computer based metal detector is that, several detection systems can be monitored from the same computer by a single attendant. A number of detection systems are interfaced to a single computer that is stationed in a suitable location. When this is done, a single person can monitor several detectors thus lowering man power requirements and therefore operation costs [14].

Still another advantage of computer based metal detector is that it can be designed to be covert in nature. In this case the magnetic sensors are concealed within the walls of the entry portal and a video camera is aimed at the sensing area to give the full view of the detector's sensing area. This eliminates the need of the screening attendant being in the immediate vicinity of the detector which is sometimes risky. Furthermore, when designed in this manner the detector is less prone to circumvention attempts since people will not be aware that theyare under surveillance. If a person carries a weapon or a threat item, automatic door closures are employed to detain him [14].

\section{THE PROPOSED SYSTEM}

The designed computer based metal detector is made up of two important parts namely hardware and software; the hardware provides the required signals to the computer in digital form. The software within the computer analyzes these signal signals giving out the desired output. The next section describes how the specific hardware and software have been designed and integrated.

\subsection{Hardware Design}

The hardware components comprises of; ten fluxgate magnetic sensors (FLC 100), USB Data Acquisition card (DAQ 2833), USB video camera (Mightex, model No. SCNCG04U), a wooden frame, two laptop computers, wireless network adapters, connecting cables, LEDs and a $5 \mathrm{~V}$ dc power supply.

The detector utilizes the earth's magnetic field to detect and classify ferromagnetic items. The magnetic sensors will detect the perturbations of earth's magnetic field caused by the presence of a ferromagnetic object in the sensing area of the detector. Figure (1) shows a screenshot of all the detector's hardware components after assembling. The sensors are mounted on a wooden frame (of height $2 \mathrm{~m}$, width $0.75 \mathrm{~m}$ and depth $0.3 \mathrm{~m}$ ), adjacent sensors being separated by a distance of $30 \mathrm{~cm}$. Each sensor is configured to generate a voltage signal with amplitude that is proportional to the magnetic field detected by the sensor. The sensors are so arranged that a subject passing through the portal may be scanned both on the right and on the left side. If a ferromagnetic item is carried, the nearest sensor to the item will detect it and the signal produced sent to the computer for analysis. The USB DAQ 2833 is in communication with each magnetic sensor.

A video camera is aimed at the sensing region of portal; it continuously monitors the passage of the subjects through the portal of the detector. Only the image of the person carrying a threat ferromagnetic item is captured. The video camera is switched to the image capture mode by the action of a software trigger. The image helps in determining the actual location of the ferromagnetic item carried by the person being screened. It is also saved in the computer for future reference and sent to the remote computer for further storage. In addition, the metal detector can be monitored by a remote computer. The hardware circuit design of the detection system is illustrated in the figure (2). It shows how the sensors are connected to the USB DAQ 2833 and the USB video camera is within the portal view. The signals from the two are taken to the server computer through USB cables and eventually transmitted to a remote computer after analysis through Data socket networking. Several parameters can be adjusted from the front panel of the detector, these are; the threshold settings for threat and non-threat items, the gain of the signals acquired, the number of channels from which the signals are acquired and the frequency of the audio alarms. 


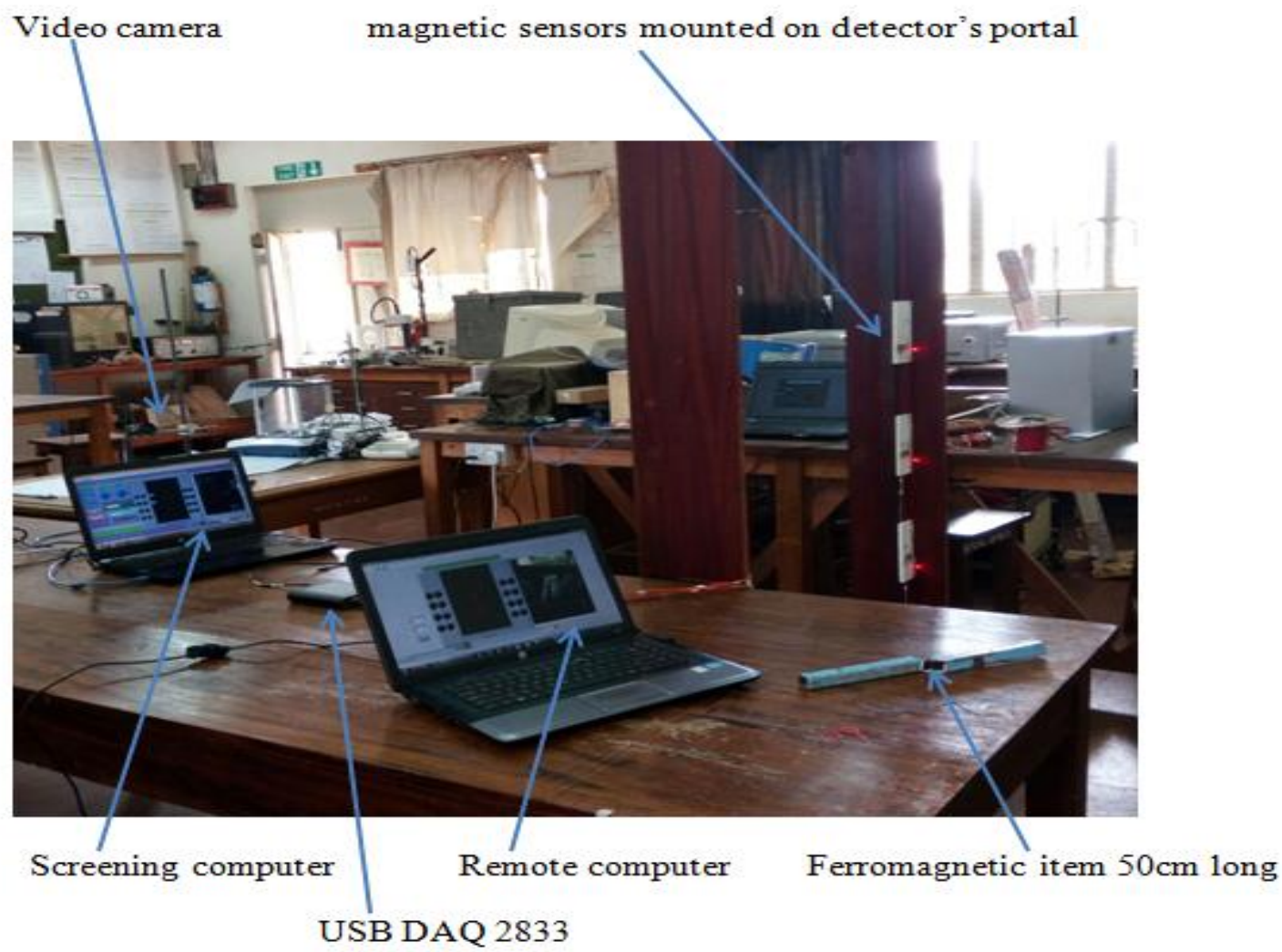

Figure 1: A screenshot showing all the assembled hardware components of the ferromagnetic metal detector.

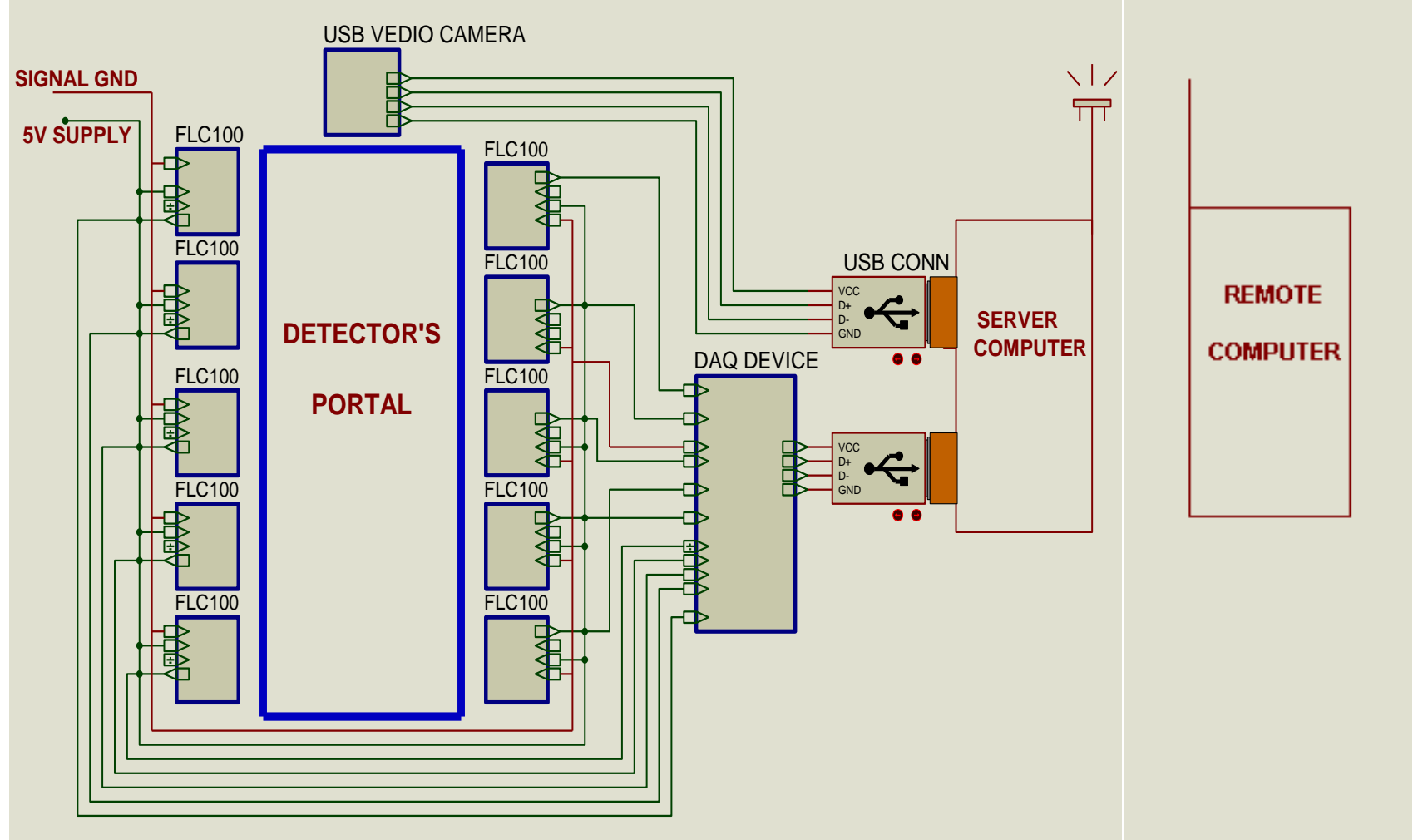

Figure 2: A schematic diagram of the hardware circuit design of the ferromagnetic metal detector

\subsection{Software Design}

LabVIEW codes were developed to perform signal acquisition from the magnetic field sensors through the USB DAQ card and image acquisition from the USB video camera. The signals acquired are then processed, analyzed and the results displayed on the computer screen. The following LabVIEW codes also called VI.s (Virtual Instruments) were designed to interface and control the metal detector from the computer.

i) The VI. for signal acquisition from the magnetic sensors. 
ii) The VI. for camera triggering and image acquisition.

iii) The VI. for recognition algorithm.

iv) The VI. for networking the screening and the remote computer.

v) The VI. for remote monitoring (in the remote computer).
3.3 The Flowchart for the System Software

When the metal detection program is run continuously, the software keeps monitoring the state of magnetic field sensors, if the sensors output signal values are greater than a set threshold, the program continues to the next level. Figure 3 illustrates the steps followed in the metal detection process.

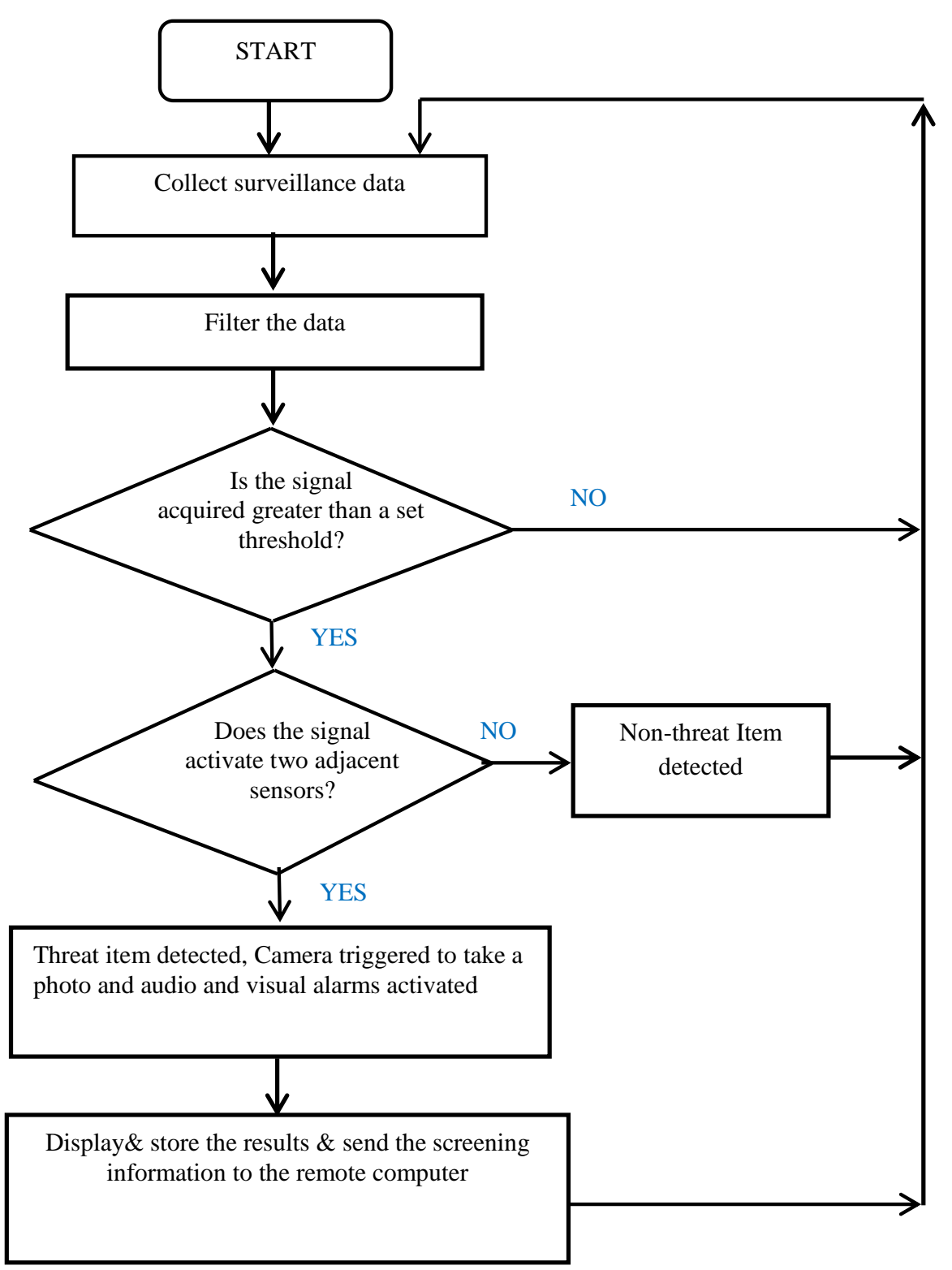

Figure 3: The flow chart for the system software design

\section{RESULTS AND DISCUSSIONS 4.1 The Effects of Changing the Sensor Orientation}

An experiment was done to study the behavior of ferromagnetic (or test) item (which measures $3 \mathrm{~cm} \times 3 \mathrm{~cm} \times$ $5 \mathrm{~cm}$ ) in earth's magnetic field. The item approaches the magnetic sensor when the sensor detection axis is oriented in
$N-S$ direction as shown in figure 4. The direction of orientation was determined by a magnetic compass. The values of magnetic field $(n T)$ measured at various distances $(\mathrm{cm})$ are recorded in table 1. The same experiment is repeated with the sensor axis being oriented in $30^{\circ}$ and $60^{\circ}$ from $N$ - S direction as shown in figure 5 and the values of magnetic field and distance recorded in table 1 . 


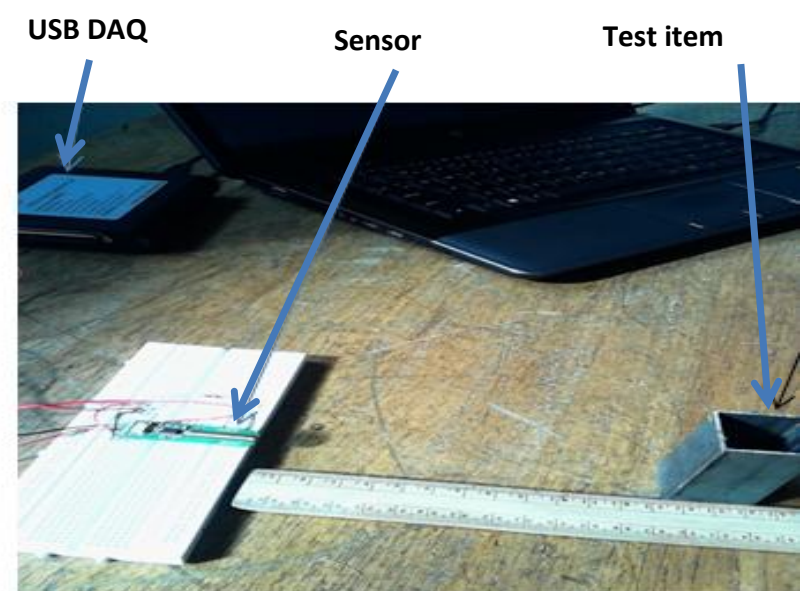

Figure 4: A ferromagnetic item approaching a magnetic sensor when the sensor axis is oriented in North-South direction

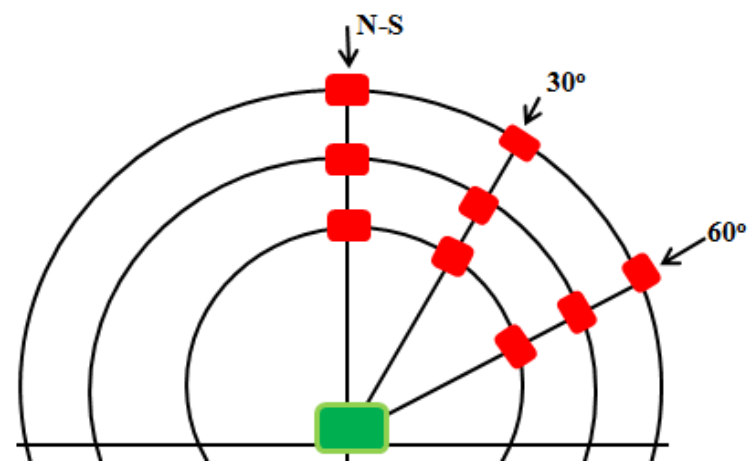

Ferromagnetic item

Magnetic sensor

Figure 5: An illustration of sensor axis being oriented in $30^{\circ}$ from $\mathrm{N}-\mathrm{S}$ direction as the ferromagnetic item approaches the sensor

The magnetic fields measured at various distances when the sensor axis lies at $\mathrm{N}-\mathrm{S}$ direction were compared to the values obtained when the sensor axis was oriented in $30^{\circ}$ and in $60^{\circ}$ from $N-S$ direction respectively. This is shown in the graphs in figure 6 and 7.

Table 1: Magnetic field $(\mathrm{nT})$ and distance $(\mathrm{cm})$ values for the test item when the sensor axis lies at N-S direction, $30^{\circ}$ and $60^{\circ}$ from $\mathrm{N}-\mathrm{S}$ direction

\begin{tabular}{|l|l|l|l|}
\hline $\begin{array}{l}\text { Magnetic } \\
\text { field, } B \text { in }(n T)\end{array}$ & $\begin{array}{l}\text { Distance } \\
\text { in } \mathrm{cm} \\
(N-S)\end{array}$ & $\begin{array}{l}\text { Distance } \\
\text { in } \mathrm{cm}\left(30^{\circ}\right. \\
\text { from } N-S)\end{array}$ & $\begin{array}{l}\text { Distance } \\
\text { in cm }\left(60^{\circ}\right. \\
\text { from } N-S)\end{array}$ \\
\hline 62.5 & 36 & 29.2 & 26 \\
\hline 125 & 20.5 & 16.5 & 15.1 \\
\hline 187.5 & 16.2 & 13.2 & 12.2 \\
\hline 250 & 14 & 11.2 & 10.7 \\
\hline 312.5 & 12.6 & 10.3 & 9.5 \\
\hline 375 & 11.6 & 9.4 & 8.7 \\
\hline 437.5 & 10.8 & 8.6 & 8.1 \\
\hline 500 & 10.2 & 8 & 7.7 \\
\hline
\end{tabular}

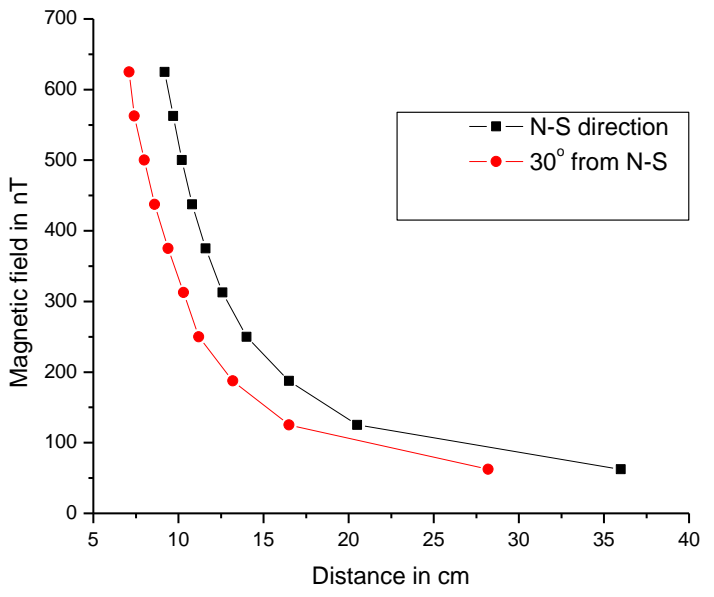

Figure 6: The graph of magnetic field $(n T)$ versus distance (cm) for the test item when the sensor axis is oriented in $30^{\circ}$ from $N-S$ and when the sensor axis lies at $N-S$ direction

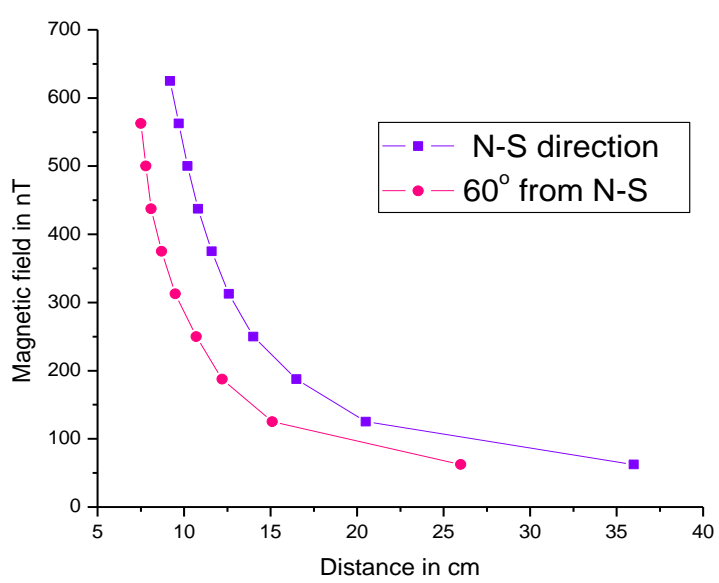

Figure 7: The graph of magnetic field (nT) versus distance $(\mathrm{cm})$ for the test item when the sensor axis is oriented in $60^{\circ}$ from $N-S$ and when the sensor axis lies at $N-S$ direction

\subsection{Placement of the Metal Detector}

From the graphs in figure 6 and figure 7, it can be noted that, the magnetic sensor measures higher values of magnetic field disturbance (caused by ferromagnetic item) when its axis of detection lies in $N-S$ direction than when lying at $30^{\circ}$ or $60^{\circ}$ from $N-S$ direction. The lowest values of magnetic field will be measured when the sensor axis lies in $E-W$ direction. The reason for this is due the nature of earth's magnetic field. Its magnetic field lines runs from earth's magnetic north pole to magnetic South Pole. So the sensor will measure the maximum disturbance of this field only when its axis of detection lies in $\mathrm{N}-\mathrm{S}$ direction. Therefore when placing a metal detector on a site, all the sensor's axes should be oriented in $N-S$ direction. When this is done, very small disturbances in earth's magnetic field can be detected thus increasing the sensitivity of the metal detector. 


\subsection{Results from the Front Panel of the Detector}

On the detector's frame, successive magnetic sensors are separated by a distance of $30 \mathrm{~cm}$. All elongated ferromagnetic items which are more than $30 \mathrm{~cm}$ long are therefore classified as 'threat items'. These items must be detected by two or more sensors and eventually cause two or more virtual LEDs to brink in red color. Apart from virtual LEDs, the other visual alarms on the computer screen are the two graphical indicators in red and green color and the words 'THREAT ITEM DETECTED' which then appears on the screen.

On the other hand if an item is compact (being less than $30 \mathrm{~cm}$ long), it will be detected by only a single sensor and it is classified as non-threat item, examples are phones, shoe shanks, belt buckles etc. When such an item is detected, the corresponding virtual LED on the front panel will brink in yellow color. The figures $8,9,10$ and 11 below shows the observations made on the front panel of the detector when the following ferromagnetic items were passed through the portal of the detector;

i) A cell phone (technop3) (non - threat item).

ii) A ferromagnetic bar $40 \mathrm{~cm}$ long (threat item).

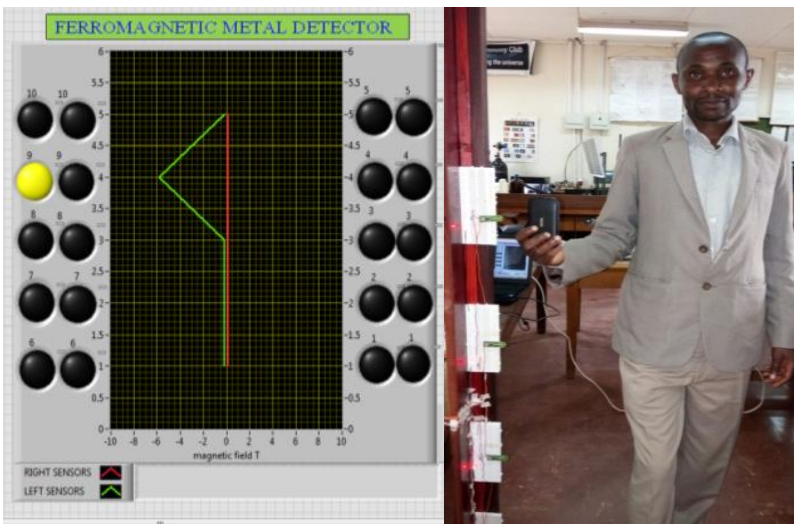

Figure 8: The front panel of the detector when a cell phone (techno P3) carried on the right hand is passed through the detector

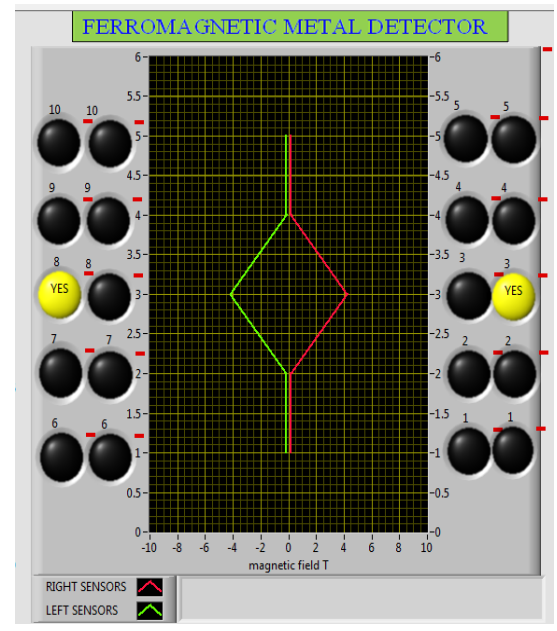

Figure 9: the front panels of the detector when two cell phones (techno P3) carried on both hands were passed through the detector

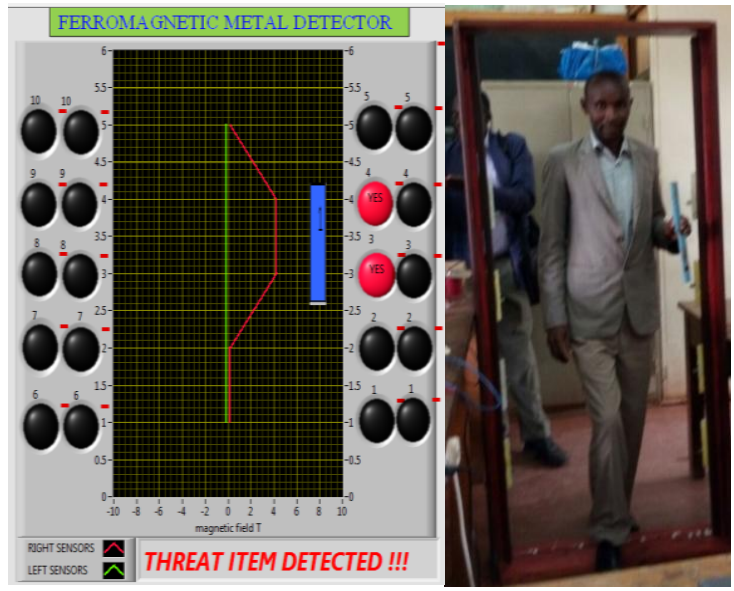

Figure 10: The front panel when a ferromagnetic bar $40 \mathrm{~cm}$ long carried on the left hand is passed through the detector

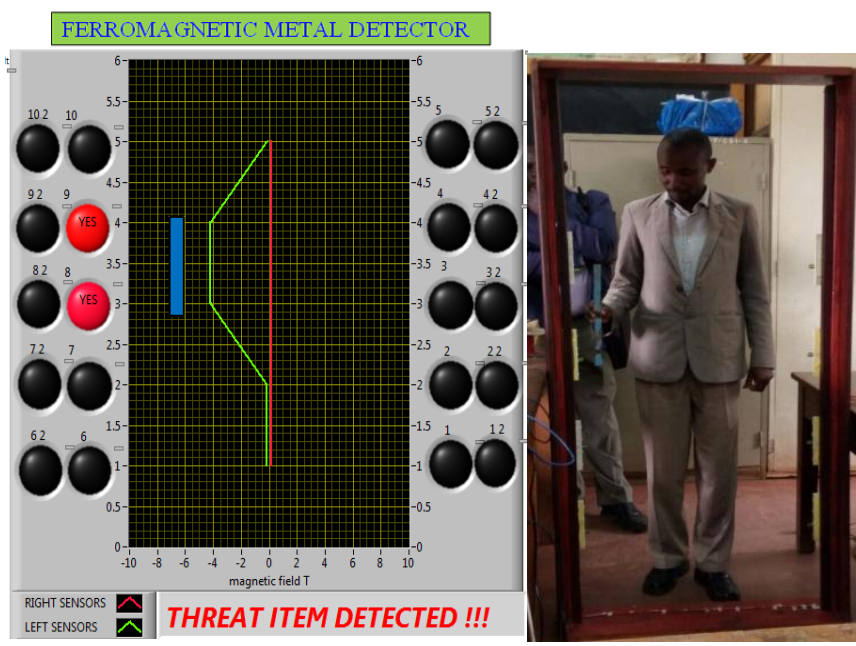

Figure 11: The front panel when a ferromagnetic bar $40 \mathrm{~cm}$ long is passed through the detector carried on right hand

\section{CONCLUSIONAND \\ RECOMMENDATION}

A computer based ferromagnetic metal detector has been designed and implemented. It has a fast settling time of approximately $1.1 \mathrm{sec}$. In addition, to lower the false alarms, a recognition algorithm has been designed in LabVIEW to discriminate between ferromagnetic items by their mass and size. All ferromagnetic items of length $30 \mathrm{~cm}$ or more classified as threat items and upon detection the audio and visual alarms are activated. The Compact items (less than $30 \mathrm{~cm}$ ) are classified as non- threat items. The allowable mass of the ferromagnetic item carried can be set as a threshold on the front panel of the detector. Two thresholds can be set i.e. for threat items and for non-threat items. Because of the improvements made, the throughput of the detector is high and it can be used for security applications with success.

As an extension to this work, a more intelligent recognition algorithm should be designed to increase the discriminating ability of the detector. This will allow the detector to recognize various ferromagnetic items even when carried in any orientation. To increase the resolution of the detector, manymagnetic sensors should be used. In addition, if sensors 
with 3-axes of detection are used, the detection volume in the sensing area will be increased.

\section{REFERENCES}

[1] V.P. Czippott, "Detection of concealed weapon using magnetic tensors tracking" final activity report, Award No. 1998-DT-CX-K002, National Institute of Justice, United states, 2001.

[2] C. Sigrist, "Metal detectors for humanitarian demining, patent search and analysis", Swiss Federal Office Education and Science, Switzerland, 2002.

[3] G.S. Burns, "In-situ vehicle classification using an ILD and a magnetoresistive sensor array", Final report, Center for Transportation Studies University of Minenesota, Duluth, United States, 2009.

[4] B. Bjerrum, "Concept development for the next generation of metal detector", Master's thesis, AllborgUniversitet, Denmark, 2008.

[5] S.P. Kalyn, "Indoor localization using magnetic fields". Dissertation for the degree of Doctor of Philosophy, University of North Texas, U.S.A, 2011.

[6] B. Sajjad, "Identification and localization on a wireless magnetic sensor network", MSc. thesis, Department of Electrical and Electronic Engineering. Middle East technical University, turkey, 2012.

[7] M.T. Yin, M.M. Myo, and M.T. Hia, "Metal Detector by Using PIC Microcontroller Interface with PC", International Journal of Scientific \& Technology Research, ISSN 2277-8616, 4: 306-311, 2015.

[8] J. Douglas, S.P. Louis and W.P. Chambers, "Magnetic screening system and network managing the same". United States Patent no. 7,898,248 B2, 2011.

[9] T. Jeffrey and K. Jim "LabVIEW for everyone $3^{\text {rd }}$ edition”, Prentice Hall. Indiana, USA, 2006.
[10] S.S. Pogula, "Developing neural network applications using LabVIEW", Master's thesis. University of Missouri-Columbia, USA, 2005.

[11] S. Breiner, "Application manual for portable magnetometers. Geometric", San Jose, California, USA, 1999.

[12] B. C. Brunschini, "A multi-disciplinary analysis of frequency domain metal detectors for humanitarian demining”. PhD. thesis, Department of Electronics and Information Processing, Vrije University Brussels, Belgium, 2002.

[13] L.G. Roybal, "Method for detecting the presence of a ferromagnetic object", Patent No. 6,150,810. United States, 2000.

[14] D. F. Fredrick, "Metal detection system and method", Patent No.7, 408,461 B2, United States, 2008.

[15] K. Govinda, S. Venkata and M. Karthikeyan, "Metal Detector for Security Checking", International Journal for Scientific Research \&Development, Issue 03ไISSN:2321-0613,4:364-366 2014

[16] G.V. Keller, "Non-obtrusive detection system and method for discriminating between concealed weapon", Patent No. 5,552,705. United States, 1996.

[17] A.Rogers. G. Nir, W. Eyal, and R.C. Tsuriel, "Ferromagnetic Mass Localization in Check Point Configuration Using a Levenberg Marquardt Algorith",Sensor9:8852-8862; doi: 10.3390/s91108852, 2009.

[18] H. Sumbul, and M. Tasdemir, "An experimental comparison of induction balance and pulse induction system", $\quad 6^{\text {th }}$ International Advanced Technologies Symposium (IATS'11) Elazığ, Turkey, 2011. 